\title{
Study of Co-electrospun Nafion and Polyaniline Nanofibers as Potential Catalyst Support for Fuel Cell Electrodes
}

\author{
Silas K. Simotwo ${ }^{1}$ and Vibha Kalra ${ }^{1}$ * \\ ${ }^{1}$ Department of Chemical and Biological Engineering, Drexel University, 3141 Chestnut \\ St., Philadelphia, PA 19104, USA. \\ *Corresponding author, email; vk99@drexel.edu
}

\begin{abstract}
Nanostructured composites of electron and proton conducting materials were fabricated via simultaneous electrospinning of Nafion and PANI solutions on a rotating collector for application as fuel cell catalyst supports. Poly (ethylene oxide) (PEO) was used as a carrier polymer to enhance chain entanglements and therefore the electrospinnability of both solutions. Dual nanofiber mats with a Nafion/PANI composition of 50/50 wt\% were prepared. Post electrospinning annealing at $150^{\circ} \mathrm{C}$ for 5 mins and densification at 500 psi was performed to enhance Nafion domain crystallinity and fiber fraction (i.e. reduce void volume), respectively. PEO was selectively removed by treatment of the electrospun mats in boiling acid solution for one hour. Conductivity of the nanofiber mats was investigated using electrochemical impedance spectroscopy and equivalent circuits were used to decouple impedance from electron and proton transfer in the composite fiber mat. The resultant dual nanofiber mat exhibited an in-plane conductivity $\left(\sigma_{=}\right)$of 0.014 and $0.023 \mathrm{~S} / \mathrm{cm}$ for electrons and protons, respectively. Through plane conductivity $\left(\sigma_{\perp}\right)$ was also studied and the dual nanofiber mat showed anisotropy for both proton and electron conductivities with $\left(\sigma_{=}\right) /\left(\sigma_{\perp}\right)=1.27$ and 1.4, respectively. Such dual nanofiber mats with percolating phases of both electron- and proton-conductors and three dimensional through-


connected inter-fiber pore structure (for gas transport) can potentially serve as excellent catalyst supports for fuel cells.

Key words: Co-electrospinning, Nanofibers, Simultaneous electron and proton conductivity, Fuel Cell Catalyst Support, Electrochemical Impedance Spectroscopy, Equivalent circuits

\section{Introduction}

Fuel cells exhibit remarkable efficiency, clean emissions and high power density, which makes them a pertinent alternative power source for applications such as electric vehicles, small portable electronics and distributed power generators [1]. Fuel cells, however, face major setbacks due to the high cost of platinum (Pt) catalyst [2] employed for electrochemical reactions. Consequently, to increase cost competitiveness of fuel cells, studies have focused on reduction of Pt loading, development of Pt alloys [3, 4] and replacement of Pt with non-precious metal catalysts [5].

To effectively reduce platinum loading, one of the key strategies to reduce Pt cost, there is a need to develop electrode architectures that will facilitate efficient transport of protons, electrons, and reactant gases to the catalyst active sites for enhanced catalyst $(\mathrm{Pt})$ utilization. In conventional fuel cells, carbon blacks (Vulcan XC-72, black pearl, etc.) are typically employed as Pt support materials due to their large surface area, and high electrical conductivity [6]. The electrode fabrication process entails the preparation of $\mathrm{Pt} /$ carbon black $(\mathrm{Pt} / \mathrm{C})$ slurry wherein Nafion is added as both a binder and proton conductor. The slurry is then painted onto a carbon paper via various techniques [7]. Electrode preparation via slurry technique, however, is likely to 
stunt catalyst accessibility due to agglomeration of carbon black nanoparticles as well as blockage of electron conduction pathways by the ionomer. Alternative nanostructured carbon materials such as carbon nanotubes [1, 8], and carbon nanofibers [9] have also been explored in place of carbon blacks. Although these high aspect ratio, nanostructured carbon support materials have demonstrated slightly enhanced electrochemical performance, their electrode preparation processes and overall electrode morphologies are akin to those of conventional $\mathrm{Pt} / \mathrm{C}$ and thus similar shortcomings are expected. Recently, effort has been made to improve electrode fabrication process in order to further enhance triple phase transport (electron, protons and gases) to catalyst active sites. Some examples of these novel designs include nanofiber-based cathodes developed by Zhang and Pintauro[10] via electrospinning of a blend of $\mathrm{Pt} / \mathrm{C}$ and Nafion, nanostructured thin films prepared by Debe et al [11] via Pt coating of organic whiskers and nanowire electrodes prepared by Alia et al [12] wherein Pt was coated onto nickel nanowires.

Electrospinning has been employed in this work to develop nanostructured electrodes that consist of well-defined co-percolating electron- and proton-conducting phases as well as excellent porosity for gas transport using Nafion, a conventional ionomer used for proton transport in fuel cells, as the proton conductor and PANI as the electron conducting material. The electrospinning technique is well documented in the literature $[13,14]$ and has been widely employed to fabricate polymeric nanofibers for application in the battery electrodes [15] and supercapacitor electrodes[16]. Both Nafion and PANI solutions have been independently electrospun in the literature. Nafion has been electrospun previously with a small fraction of carrier polymers such as PEO [17] [18, 19], poly(vinyl) alcohol [20] and PVA [21]. Membranes based on electrospun Nafion have demonstrated better electrochemical performance than solution cast membranes [22]. PANI, another conducting polymer, has previously demonstrated 
good applicability as a catalyst support material [23-25] due to its high electrical conductivity, large surface area and favorable interaction with Pt [26]. PANI has also been electrospun with aid of carrier polymers for application in supercapacitors [27], sensors/actuators [28] and thermos-electrics [29]. However, these works on PANI electrospinning use a large fraction of the insulating carrier polymer (>20 wt \%).

In this paper, we report the fabrication of nanostructured Nafion/PANI composite architecture via a single-step, simultaneous electrospinning of Nafion/PEO and PANI/PEO blend solutions. PEO was added as carrier polymer to both mixtures to enhance electrospinnability of the solutions. Both Nafion and PANI solutions are not electrospinnable by themselves due to poor dispersion of these polymers in most solvents $[19,27]$. The fabricated dual nanofiber mat possesses a three-dimensional, porous, interconnected non-woven network of both electron- and proton-conducting nanofibers making it suitable for application in the fuel cells electrodes where facile accessibility of the triple phase boundary is critical. Prior to preparation of the nanofiber composite mat, preliminary electrospinning and characterization studies were conducted on individual Nafion and PANI systems to understand electrospinning conditions and properties of individual mats. Sole Nafion and PANI nanofibers mat were prepared with PEO content of 2 and $10 \mathrm{wt} \%$, respectively. These solutions were then electrospun separately and simultaneous onto a single rotating collector to give dual Nafion/PANI nanofiber mats. Fabricated nanofiber mats were subjected to annealing, densification and treatment in boiling $\mathrm{H}_{2} \mathrm{SO}_{4}$ and DI water. Electrochemical impedance spectroscopy (EIS) showed presence of independent charge transfer channels in the composite fiber mat as illustrated by multiple arcs in the Nyquist plot spectra. Nafion/PANI nanofibers mat subjected to annealing, densification and acid treatment showed electrical and proton conductivity of 0.014 and $0.023 \mathrm{~S} / \mathrm{cm}$, respectively. 


\section{Experimental Method}

\subsection{Materials}

Nafion powder was prepared from LIQUION 1115 solution purchased from Ion Power Inc. by evaporating the water/alcohol solvents in the fume hood for 72 hours and then drying the polymer precipitate in a convection oven at $60^{\circ} \mathrm{C}$ for $45-60$ minutes. Polyethylene oxide (2000kDa), polyaniline (100kDa), camphorsulphonic acid (98\%), and 2-propanol (99.5\%) were obtained from Sigma-Aldrich. Chloroform (ACS grade, 99.8\%) was obtained from Alfa-Aesar.

\subsection{Nanofiber Fabrication}

For preparation of electrospinnable Nafion solutions, Nafion powder and PEO were dissolved in 2-propanol/water mixture $(2: 1 \mathrm{wt} / \mathrm{wt})$ under gentle stirring for 4-6 hours. Nafion concentration in the solution was $\sim 42 \mathrm{mg} / \mathrm{ml}$. For preparation of polyaniline (PANI) solutions, first PANI and camphorsulfonic acid (CSA) (1:1.29 wt/wt ratio) were dissolved in chloroform and ultrasonicated at room temperature for 15 minutes. The ultrasonicated solution was then stirred overnight also at room temperature. PEO was then added to CSA-doped PANI and the solution was further stirred for 6 hours. The solution was then left overnight without stirring. PANI concentration in chloroform was $\sim 10 \mathrm{mg} / \mathrm{ml}$. For all electrospinning experiments, the distance between the tip of the needle (22 gauge needle spinneret from Hamilton Company) and the grounded collector was fixed at 5-6", the applied voltage was kept at 5-7 kV and the relative humidity was maintained below $20 \%$ to obtain a stable Taylor cone. The solution flow rates for Nafion and PANI streams were $\sim 0.30$ and $1.25 \mathrm{ml} / \mathrm{hr}$, respectively. While the individual Nafion and PANI nanofiber mats were collected on a planar substrate, a cylindrical drum collector, 
rotating at $60 \mathrm{rpm}$, was employed for the dual system. A schematic of the dual electrospinning set up is shown in figure 1. For ease of reference in the subsequent discussions, as electrospun Nafion/PEO (98/2 wt \%), PANI/PEO (90/10 wt\%) and Nafion/PANI/PEO (47.5/47.5/5) nanofiber mats will be referred to as Nafion-98, PANI-90 and Nafion-PANI, respectively.

The fabricated samples were subjected to post electrospinning vacuum annealing at $150^{\circ} \mathrm{C}$ for 5 minutes, followed by compression at $\sim 500$ psi and finally treatment in boiling acid (1M $\mathrm{H}_{2} \mathrm{SO}_{4}$ ) for $1 \mathrm{~h}$ and in DI water for another $1 \mathrm{~h}$. For ease of reference, prefix AN, $\mathrm{C}$ and $\mathrm{T}$ will be added to the above defined sample nomenclature to indicate annealed, compressed, and acid/water treated samples. Combination such as AN/C will refer to samples both annealed and compressed.

\subsection{Nanofiber Characterization}

Scanning electron microscopy (SEM) (Zeiss Supra 50VP) was used to study the external morphology of as-electrospun and processed Nafion, PANI and Nafion/PANI nanofiber mats. Fourier transform infrared spectroscopy (FTIR) (Varian Excalibur FTS-3000, range of 4000-800 $\mathrm{cm}^{-1}$ ), X-ray Diffraction (XRD) (Rigaku SmartLab, X-ray diffractometer, $\mathrm{Cu} \mathrm{K} \alpha$, scanning range $5-40^{\circ}$ and step size of $0.02^{\circ}$ ) and thermogravimetric analysis (TGA Q50 analyzer, range 20$800^{\circ} \mathrm{C}$ with step size of $10^{\circ} \mathrm{C}$ in argon atmosphere) were used to probe the chemical structure, crystallinity and composition/thermal stability of the nanofiber samples. XRD Peaks were fitted using Pearson VII distribution function in OriginPro software.

\subsection{Water uptake and Conductivity}

Weights of dry nanofiber mats were first measured using mass balance (Ohaus DV215CD). The dry samples were then soaked in milli-Q water (18.2 M $\Omega . c m)$ for $48 \mathrm{hrs}$. Samples were 
removed from water bath, quickly blotted with tissue paper to remove surface water and weighed again. Mass swelling was then calculated as follow;

$$
\text { Water uptake }=\frac{W_{w e t}-W_{d r y}}{W_{d r y}} * 100 \%
$$

where w denotes the sample weight.

In-plane conductivity $\left(\sigma_{=}\right)$of the samples was studied via electrochemical impedance spectroscopy (EIS) where a four-point probe conductivity cell made of a Teflon block with four platinum wires mounted on it was used. Prior to impedance studies, the samples (with exception of PANI-90) were soaked in distilled water at room temperature for 48 hours. Gamry 3000 reference potentiostat was used to generate impedance spectra. Expression 1 below was employed to compute sample conductivity.

$$
\sigma=\frac{L}{R * W * t}
$$

where $\sigma$ is the sample conductivity $(\mathrm{S} / \mathrm{cm}), \mathrm{L}$ is the distance between the inner probes of a 4 probe cell configuration, $\mathrm{w}$ and $\mathrm{t}$ represents the sample width and thickness respectively. Through plane conductivity $\left(\sigma_{\perp}\right)$ of the annealed, compressed and treated nanofibers mat was also established using stainless steel current collectors (diameter $1.26 \mathrm{~cm}$ ). For through plane, L in expression 1 above represent thickness of the nanofiber mat while $\left(\mathrm{w}^{*} \mathrm{t}\right)$ is the area of the current collector.

\section{Results and discussion}

\subsection{Morphology of electrospun nanofibers}


SEM was used to probe the morphology of electrospun Nafion-98, PANI-90 and NafionPANI nanofiber mats. Figure 2 (a) shows the SEM micrograph of as electrospun Nafion-98 fiber mat. Nanofibers exhibited smooth morphologies without any beads. The bead-free morphology is attributed to the presence of PEO which is thought to impart the necessary entanglement in the polymer blend solution and therefore enhance its viscoelasticity for better electrospinnability [18]. The diameter of the electrospun nanofibers was in the range of 200-400nm. Nafion-98 nanofiber mats were subjected to post electrospinning annealing at $150^{\circ} \mathrm{C}$ under vacuum for 5 minutes and densified at 500 psi. Annealing was observed to cause welding of intersecting Nafion nanofibers, a phenomena attributed to Nafion plasticization above its glass transition temperature of $\sim 107^{\circ} \mathrm{C}[30]$.

PANI is a promising conducting polymer for applications in electrodes for energy devices. PANI is an extremely difficult polymer to process due to its poor solubility/dispersion in most organic solvents. Moreover, PANI solutions lack the requisite viscosity for electrospinning attributed to its shorter and rigid polymer chains. Some work has been reported on electrospinning of PANI by blending it with a carrier polymer such as PEO which alleviates the lack of chain entanglement issue. MacDiarmid et al [31] reported fabrication of PANI/PEO blend with w/w composition of 50/50. Most of the PANI nanofibers reported, however, have high content of the non-conductive carrier polymers (>20 wt $\%)[32,33]$.

In this work, we report fabrication of PANI nanofibers with low PEO content of only 10 wt\% by use of a relatively higher molecular weight of PEO (2000 kDa). PANI purchased in the emaraldine-base form was first doped with camphorsulfonic acid to protonate the imine groups. Emaraldine PANI exists in a semi-reduced/oxidized state and its protonation with aqueous acids have been shown to increase its conductivity remarkably due to increased $\pi$-delocalization of the 
polymer backbone[34-36]. Even though dispersion of PANI in chloroform as high as $2 \mathrm{wt} \%$ has been reported [31], we observed that it was difficult to electrospin PANI solution with more than $1 \mathrm{wt} \%$ in chloroform. As a result, chloroform solution with $0.67 \mathrm{wt} \%$ PANI concentration was used for electrospinning in this work. SEM micrograph in Figure 3 shows the PANI-90 nanofibers morphology characterized by nanofibers that are free of beads. The nanofibers diameter were in $506 \pm 115 \mathrm{~nm}$ (calculated using imageJ software for 100 nanofibers). The electrospun PANI-90 nanofibers were also subjected to annealing at $150^{\circ} \mathrm{C}$ under vacuum for 5 mins. Prior thermogravimetric studies under inert atmosphere (figure S2) had confirmed thermal stability of PANI at these conditions. From SEM micrographs, there were no observable morphological changes on PANI-90 nanofibers after annealing.

Fabrication of the dual Nafion/PANI system entailed co-electrospinning of Nafion/PEO (98/2 wt $\%$ ) and PANI/PEO (90/10wt \%). For proper intertwining of Nafion and PANI nanofibers, a rotating drum substrate collector was used. Low rotational speed of the motor (60 rpm) was necessary since fibers have been known to align at higher speeds [29, 37]. For the targeted application, it is critical to achieve a random orientation of nanofibers to maintain a three-dimensional non-woven structure to facilitate gas diffusion and for formation of triple phase surfaces. SEM micrograph in Figure 4 (a) shows the nanostructured fiber morphology of the Nafion-PANI system. The nanofibers exhibit an excellent smooth morphology. SEM EDS shown in supporting information section (figure S1) was employed to map the distribution of fluorine and nitrogen, elements unique to Nafion and PANI respectively. The images show even distribution of both phases. The nanofiber mats were also subjected to post electrospinning annealing at $150^{\circ} \mathrm{C}$ under vacuum. Figure 4(b) shows the morphology of annealed dual fiber mat. Nanofibers are observed to be intersecting/welding, an aforementioned phenomena 
encountered when annealing Nafion-98 nanofibers. Figure 4 (c) shows annealed Nafion-PANI nanofiber mat further densified at 500 psi. Densification of the nanofibers was aimed at increasing inter-fiber contact as well as well as reducing the void volume. Moreover, membrane electrode assembly fabrication process entails the application of such compression conditions [1, 38] and therefore this treatment was necessary to gauge the ability of the fabricated nanofiber mats to withstand such conditions. Figure 4 (d) shows the Nafion-PANI nanofibers both annealed, compressed and treated in sulfuric acid as discussed in section 2. Solution cast Nafion membranes are typically subjected to pretreatment for $1 \mathrm{hr}$ in boiling $\mathrm{H}_{2} \mathrm{SO}_{4}$ and $1 \mathrm{hr}$ in boiling DI water. The pretreatment process is aimed at enhancing protonation and hydration of Nafion's sulfonic groups [39]. The electrospun Nafion-PANI fiber mat was subjected to similar treatment after its annealing. This treatment process additionally aids in selective dissolution of PEO [40]. The Nafion-PANI nanofiber morphology is retained, indicating presence of continuous phases of both Nafion and PANI in their respective blends with PEO.

\subsection{XRD and FTIR characterization}

Figure 5 shows the XRD spectra for as-electrospun and annealed Nafion-98 while figure 6 shows XRD pattern for PANI-90 and Nafion-PANI. The XRD spectrum of commercial Nafion-212 is provided for reference purposes. Crystallinity of Nafion is essential in controlling properties such as density, hardness/brittleness, and coefficient of expansion [41]. A correlation between Nafion crystallinity and its conductivity has also been observed [37]. For Nafion, the Xray beam is diffracted by the crystalline region according to Bragg's law and diffuse scattered by the amorphous region. While the Nafion crystalline phase is represented by an XRD peak at an angle of $\sim 18^{\circ}$, the amorphous phase is observed as a broad halo at a diffraction angle of $\sim 16.5^{\circ}$ due to diffuse scattering of the incident X-ray beam [30,41]. As shown in figure 5, the XRD 
pattern of Nafion-212 membrane shows a crystalline peak at a diffraction angle of $\sim 17.5$ whereas for the as-electrospun Nafion-98 sample, we largely observe a broad peak around $\sim 17^{\circ}$ where the peak due to crystallinity isn't as clear as that observed for Nafion-212 possibly due to large interference from the scattering of $\mathrm{x}$-ray beam by the amorphous region. Upon annealing for 5 minutes, we see a slight growth of the peak close to $17.5^{\circ}$ which we believe indicates increase in crystallinity of Nafion nanofibers. Further annealing of the electrospun nanofibers for $2 \mathrm{~h}$ at the same temperature led to further growth of the peak and an XRD spectrum much similar to that of Nafion-212. The XRD peaks have been fitted using Pearson VII distribution function in OriginPro software. Ballengee et al [30] observed that longer annealing (up to 2hrs) of electrospun Nafion nanofibers led to further growth in crystalline peak. However, they observed a drop in conductivity for an annealing time greater than 15 mins. PANI-90 XRD peaks were at $2 \theta=\sim 15^{\circ}, 20^{\circ}$ and $25^{\circ}$. Characteristic PANI peaks at $2 \theta=\sim 15^{\circ}$ and $2 \theta=\sim 25^{\circ}$ are attributed to the periodicity of the repeat unit of PANI chain and periodicity parallel to the polymer chain backbone [29]. An overlap is observed between PANI peak at $2 \theta=\sim 15^{\circ}$ and Nafion crystallinity peak at $16-18^{\circ}$, thus the extent of Nafion crystallinity in the dual mat is difficulty to decipher without any ambiguity.

FTIR absorbance spectra shown in figure 7 were recorded in the 800 to $4000 \mathrm{~cm}^{-1}$ range at $25^{\circ} \mathrm{C}$ to investigate the chemical structure of electrospun nanofibers. Figure 7 shows the FTIR spectra for dry Nafion-98 (a), PANI-90 (b) and Nafion-PANI (b) nanofibers. FTIR spectra of Nafion-212 was also obtained for reference. Characteristic Nafion peaks at; 1145 and $1200 \mathrm{~cm}^{-1}$ associated with symmetric and asymmetric stretching of $-\mathrm{CF}_{2}$ groups, respectively, 980 (stretching mode of C-O-C group), 1060 (vibration of $-\mathrm{SO}_{3}$ group) and $1410 \mathrm{~cm}^{-1}$ (vibration of $\mathrm{S}=\mathrm{O})$ were observed [42-44] for both Nafion-212 and the Nafion nanofiber mats. Weak water 
bands around 3460 and $1600-1700 \mathrm{~cm}^{-1}$ [42] were also observed in all systems indicating absorption of moisture from the atmosphere. No perceptible shift was observed in the location of typical Nafion bands for both as electrospun and annealed samples. The presence of PEO in a Nafion matrix is thought to lead to interaction between the ether oxygen of PEO and $\mathrm{H}$ atom of the Nafion sulfonic group via hydrogen bonding [45]. The IR signal from such interaction is expected to overlap with the water bands at around $3500 \mathrm{~cm}^{-1}$. To better understand this, we compared the FTIR peak intensities (at $3460 \mathrm{~cm}^{-1}$ ) of Nafion-98 with those of commercial Nafion-212. FTIR intensity ratio, $I / I_{0}$, where $I$ is the intensity of Nafion- 98 and $I_{0}$ is the intensity of Nafion-212 was calculated to be $\sim 0.26$, which can be corrected to 1.13 to account for the low Nafion fraction (20-25\%) in fiber mats compared to films. $I / I_{0}$ ratio of greater than 1 could thus be potentially due to PEO interaction with Nafion sulfonic, group which might impede Nafion conductivity.

FTIR spectra of dual Nafion-PANI nanofiber mat shows characteristics PANI and Nafion peaks. The peak at $1060 \mathrm{~cm}^{-1}$ associated with symmetric stretching mode of $-\mathrm{SO}_{3}$ group is slightly shifted to $1050 \mathrm{~cm}^{-1}$. The shift could be due to the induced polarization of the S-O dipole by the electrostatic field of the adjacent protonated imine group of PANI [46].

\subsection{Impedance study}

EIS was employed to characterize the impedance of the nanofiber mats. Prior to obtaining the EIS data, fiber mats were equilibrated in distilled water for at least $48 \mathrm{~h}$. The proton conductivity of Nafion occurs through dissociation of protons from $-\mathrm{SO}_{3} \mathrm{H}$ ionic clusters. These protons are then transported across water and $-\mathrm{SO}_{3}{ }^{-}$ionic fixed groups [21] and thus the necessity of hydration of the fiber mats. 
Table 1 shows the conductivity summary of the prepared nanofiber mats. Impedance data for Nafion-98 and PANI-90 mats was obtained from Nyquist plots shown in the supporting information (figure S3). As electrospun Nafion-98 showed a proton conductivity of $0.021 \mathrm{~S} / \mathrm{cm}$. The fiber fraction of this nanofiber mat was ascertained to be $\sim 23 \%$ (supporting info table S-1). Thus the corrected conductivity of Nafion-98 is approximately $0.091 \mathrm{~S} / \mathrm{cm}$. This value compares favorably with that of pristine Nafion film which has proton conductivity of $0.10 \mathrm{~S} / \mathrm{cm}$ [39]. PANI-90 showed an electrical conductivity of $0.078 \mathrm{~S} / \mathrm{cm}$ from EIS spectra. This value was confirmed through dc polarization experiments where a small dc potential was applied and the corresponding steady current was recorded. The conductivity value recorded is comparable to that reported by MacDiarmid et al [31] of $0.10 \mathrm{~S} / \mathrm{cm}$, where they electrospun a PANI-PEO at 50/50wt $\%$, but obtained fibers with diameters in the micron scale. Figure 8 shows the experimental and fitted impedance data for Nafion-PANI dual nanofiber mats. The Nyquist spectrum is characterized by two arcs, representing the parallel combination of ionic impedance and geometric capacitance at higher frequency, and electronic impedance and geometric capacitance at lower frequency [47]. Equivalent circuits (discussed below) were used to model the EIS experimental data to decouple the protonic and electronic impedance values. As electrospun Nafion-PANI nanofibers showed proton and electron conductivity of 0.0078 and $0.0046 \mathrm{~S} / \mathrm{cm}$, respectively. Both electrical and proton conductivity are reduced in the dual nanofiber system compared to that of individual PANI-90 and Nafion-98 systems due to the lower fraction of both the PANI and Nafion phase in the dual fiber mat.

Annealing was observed to improve the conductivity of all samples with the exception of PANI-90 nanofiber mat (see table 1). Conductivity of annealed Nafion-98 was $0.026 \mathrm{~S} / \mathrm{cm}$, which represents a $\sim 20 \%$ increase from that of as-electrospun mat. Annealing of Nafion-PANI 
also resulted in a similar enhancement of proton conductivity and about $9 \%$ increase in electrical conductivity. It was experimentally observed that annealing of nanofibers resulted in increase in the density of Nafion-based nanofibers implying increased compactness of the nanofibers (table S-1). Nafion nanofibers were also seen to weld together (also see AN Nafion-98 SEM micrographs) from SEM images upon annealing, which possibly resulted in improved fiber-fiber contact and higher charge transport. Indeed densification of nanofibers at 500 psi led to increase in both electrical and proton conductivity. Ballengee et al [30] observed similar effect on proton conductivity upon annealing of Nafion nanofibers for less than 15 mins at $150^{\circ} \mathrm{C}$ which they attributed to increased water uptake of the nanofibers. However, the water uptake for our annealed electrospun nanofibers is less than that of as-electrospun nanofibers. Annealing was also observed to increase the extent of crystallinity especially for Nafion-98 as shown in figure 5 (a). Increase in ordered structure of annealed materials could also contribute to the elevation of proton conductivity. Annealed and compressed Nafion-PANI nanofibers were subjected to treatment in boiling $1 \mathrm{M} \mathrm{H}_{2} \mathrm{SO}_{4}$ for $1 \mathrm{~h}$ and DI water for $1 \mathrm{~h}$. The treatment was intended to enhance hydration and protonation of the sulfonic group of Nafion. The treatment process has also been to shown to aid PEO removal from Nafion nanofibers [40]. Presence of insulating PEO in Nafion matrix is expected to suppress proton conductivity. Furthermore, PEO is chemically unstable in the presence of peroxides/hydroxides. It was observed that the annealing, compression and treatment of dual Nafion nanofibers in acidic/water media led to a significant increase in both proton (three-fold) and electrical conductivity (two-fold). PEO removal was confirmed via TGA (figure S2).

Since through plane conductivity set up gives a true representation of the dominant pathway travelled by the electrons and protons during fuel cell operation, we measured the 
through plane conductivity of the final dual nanofiber mat. For AN/C/T Nafion-PANI nanofiber mat, through plane proton and electron conductivities were measured as 0.018 and $0.010 \mathrm{~S} / \mathrm{cm}$ resulting in a $\sigma_{\Xi} / \sigma_{\perp}$ ratio of 1.4 and 1.27 , respectively. This anisotropy in the conductivity of our electrospun nanofibers can be hypothesized to be due to polymers' molecular/ionic domain aligning along the fiber axis as a result of extensional strain during electrospinning process [19]. Thus in-plane conductivity is slightly favored. The slight increase in through plane resistance might also be attributed to interference from mat/current collector resistance which is known to be more pronounced for through plane due to a large membrane/current collector contact area [48]

Table 1: Conductivity and water uptake of electrospun nanofiber mats.

\begin{tabular}{llll}
\hline Sample & Proton conductivity & Electron Conductivity & Water Uptake \\
& $(\mathbf{S} / \mathbf{c m})$ & $(\mathbf{S} / \mathbf{c m})$ & $(\Delta \mathbf{w t} \%)$ \\
\hline PANI-90 & & 0.0780 & $83 \pm 1.2$ \\
AN PANI-90 & & 0.0760 & $81 \pm 2.3$ \\
Nafion-98 & 0.0210 & & $40 \pm 3.2$ \\
AN Nafion-98 & 0.0260 & & $36 \pm 2.7$ \\
Nafion-PANI & 0.0078 & 0.0043 & $65 \pm 2.5$ \\
AN Nafion-PANI & 0.0094 & & $56 \pm 2.1$ \\
AN/C/T Nafion-PANI & 0.0230 & 0.0050 & $52 \pm 2.8$
\end{tabular}


For AN/C/T, through plane set up gave conductivity of 0.018 and $0.010 \mathrm{~S} / \mathrm{cm}$ for proton and electrons, respectively.

Figure 9 shows the equivalent circuit used to decouple the proton and electron conductivity values provided above from the measured impedance data. The circuit was built using a Gamry 3000 reference model builder. The dual nanofiber mats are expected to contain co-percolating channels for electron and proton flow, thus a parallel combination of electronic $\left(Z_{\mathrm{e}}\right)$ and protonic (represented as $\mathrm{Z}_{\mathrm{p}}$ ) impedance units is proposed, both in parallel with the overall geometric capacitance, $\mathrm{C}_{\mathrm{b}}$. The capacitor elements are modeled as non-ideal capacitors [49]. Since the electrodes used (Pt electrodes) are expected to be blocking to ionic flow at the sample/Pt electrode contact interface [48, 50], a capacitive element, $\mathrm{C}_{\mathrm{int}}$, is added in series to the ionic impedance element. Any impedance to electron flow at the sample/Pt electrode contact is treated as negligible. There is also an initial resistance observed for the nanofiber mats. The resistance is represented as, $R_{s}$, and can be attributed to the resistance due to connecting wires.

\section{Conclusion}

Composite Nafion/polyaniline nanofiber mats were fabricated and studied with potential application as catalyst supports in fuel cells electrodes. The electrospun dual nanofiber mats were subjected to post electrospinning processing - annealing, compression and treatment in acidic/DI water media — in order to improve its conductivity, mechanical strength and chemical stability. Electrochemical impedance spectroscopy (EIS) was used to study conductivity properties of the dual fiber mats and an equivalent circuit was used to decouple the proton and electron conductivities. EIS spectra was characterized by two semi-circles indicating that the 
composite fiber mat possessed percolating channels for both electron and proton conduction, a requisite property for fuel cell catalyst supports. The annealed/compressed/treated dual fiber mat exhibited a proton and electron conductivity of up to 0.023 and $0.014 \mathrm{~S} / \mathrm{cm}$ respectively. The fabricated fiber mat with the presence of percolating channels for electron and proton conduction and three-dimensional inter-fiber macrospores (for gas and water transport) can serve as an efficient freestanding catalyst support for PEMFCs. Future work on incorporation of CNTs within the PANI nanofibers to bolster its mechanical stability/ electrical conductivity as well introduction of platinum/catalyst nanoparticles via electrodeposition on a freestanding nanofiber mat is underway.

\section{Acknowledgement}

We would like to thank National Science Foundation (award number: CBET 1150528) for funding. We are also grateful to Drexel University Centralized Research Facility for the use of their characterization equipment. 


\section{References}

[1] B. Fang, M. Kim, J.H. Kim, M.Y. Song, Y. Wang, H. Wang, D.P. Wilkinson, J.-S. Yu, High Pt loading on functionalized multiwall carbon nanotubes as a highly efficient cathode electrocatalyst for proton exchange membrane fuel cells, Journal of Materials Chemistry, 21 (2011) 8066-8073.

[2] G. Wu, K.L. More, C.M. Johnston, P. Zelenay, High-Performance Electrocatalysts for Oxygen Reduction Derived from Polyaniline, Iron, and Cobalt, Science, 332 (2011) 443-447.

[3] A. Marcu, G. Toth, R. Srivastava, P. Strasser, Preparation, characterization and degradation mechanisms of PtCu alloy nanoparticles for automotive fuel cells, Journal of Power Sources, 208 (2012) 288-295.

[4] M. Oezaslan, F. Hasche, P. Strasser, Oxygen Electroreduction on PtCo3, PtCo and Pt3Co Alloy Nanoparticles for Alkaline and Acidic PEM Fuel Cells, Journal of The Electrochemical Society, 159 (2012) B394-B405.

[5] R. Othman, A.L. Dicks, Z. Zhu, Non precious metal catalysts for the PEM fuel cell cathode, International Journal of Hydrogen Energy, 37 (2012) 357-372.

[6] M. Hezarjaribi, M. Jahanshahi, A. Rahimpour, M. Yaldagard, Gas diffusion electrode based on electrospun Pani/CNF nanofibers hybrid for proton exchange membrane fuel cells (PEMFC) applications, Applied Surface Science, 295 (2014) 144-149.

[7] S. Litster, G. McLean, PEM fuel cell electrodes, Journal of Power Sources, 130 (2004) 61-76.

[8] J.M. Tang, K. Jensen, M. Waje, W. Li, P. Larsen, K. Pauley, Z. Chen, P. Ramesh, M.E. Itkis, Y. Yan, R.C. Haddon, High Performance Hydrogen Fuel Cells with Ultralow Pt Loading Carbon Nanotube Thin Film Catalysts, J. Phys. Chem, 111 (2007) 17901-17904.

[9] J. Park, Y. Ju, S. Park, H. Jung, K. Yang, W. Lee, Effects of electrospun polyacrylonitrile-based carbon nanofibers as catalyst support in PEMFC, Journal of Applied Electrochemistry, 39 (2009) 1229-1236.

[10] M. Brodt, R. Wycisk, P.N. Pintauro, Nanofiber Electrodes with Low Platinum Loading for High Power Hydrogen/Air PEM Fuel Cells, Journal of the Electrochemical Society, 160 (2013) F744-F749.

[11] M.K. Debe, A.K. Schmoeckel, G.D. Vernstrom, R. Atanasoski, High voltage stability of nanostructured thin film catalysts for PEM fuel cells, Journal of Power Sources, 161 (2006) 1002-1011.

[12] S.M. Alia, B.A. Larsen, S. Pylypenko, D.A. Cullen, D.R. Diercks, K.C. Neyerlin, S.S. Kocha, B.S. Pivovar, Platinum-Coated Nickel Nanowires as Oxygen-Reducing Electrocatalysts, ACS Catalysis, 4 (2014) 11141119.

[13] Z. Huang, Y.Z. Zhang, M. Kotaki, S. Ramakrishna, A review on polymer nanofibers by electrospinning and their applications in nanocomposites, Composites Science and Technology, 63 (2003) 2223-2253.

[14] A. Baji, Y. Maia, S.-C. Wong, M. Abtahi, P. Chen, Electrospinning of polymer nanofibers: Effects on oriented morphology, structures and tensile properties, Composites Science and Technology, 70 (2010) 703-710.

[15] K. Hwang, B. Kwon, H. Byun, Preparation of PVdF nanofiber membranes by electrospinning and their use as secondary battery separators, Journal of Membrane Science, 378 (2011) 111-116.

[16] C. Tran, V. Kalra, Fabrication of porous carbon nanofibers with adjustable pore sizes as electrodes for supercapacitors, J. Power Sources, 235 (2013) 289-296.

[17] A. Laforgue, L. Robitaille, A. Mokrini, A. Ajji, Fabrication and Characterization of Ionic Conducting Nanofiber, 292 (2007) 1229-1236.

[18] J.B. Ballengee, P.N. Pintauro, Morphological Control of Electrospun Nafion Nanofiber Mats, Journal of The Electrochemical Society, 158 (2011) B568.

[19] B. Dong, L. Gwee, D. Salas-de la Cruz, K.I. Winey, Y.A. Elabd, Super proton conductive high-purity nafion nanofibers, Nano letters, 10 (2010) 3785-3790. 
[20] H. Chen, J.D. Snyder, Y.A. Elabd, Electrospinning and Solution Properties of Nafion and Poly(acrylicacid), Macromolecules 41 (2008) 128-135.

[21] S. Mollá, V. Compañ, Polyvinyl alcohol nanofiber reinforced Nafion membranes for fuel cell applications, Journal of Membrane Science, 372 (2011) 191-200.

[22] J. Choi, K.M. Lee, R. Wycisk, P.N. Pintauro, P.T. Mather, Nanofiber Network lon-Exchange Membranes, Macromolecules, , 41 (2008) 4569-4572.

[23] J. Choi, Y. Kim, J. Lee, K. Cho, H. Jung, J. Park, I. Park, Y. Sung, A polyaniline supported PtRu nanocomposite anode and a Pd-impregnated nanocomposite Nafion membrane for DMFCs, Solid State lonics, 176 (2005) 3031-3034.

[24] Z. Chen, L. Xu, W. Li, M. Waje, Y. Yan, Polyaniline nanofibre supported platinum nanoelectrocatalysts for direct methanol fuel cells, Nanotechnology, 17 (2006) 5254-5259.

[25] B. Rajesh, K.R. Thampi, J.M. Bonard, H.J. Mathieu, N. Xanthopoulos, B. Viswanathan, Nanostructured Conducting Polyaniline Tubules as Catalyst Support for Pt Particles for Possible Fuel Cell Applications, Electrochemical and Solid-State Letters, 7 (2004) A404.

[26] S. Chen, Z. Wei, X. Qi, L. Dong, Y.G. Guo, L. Wan, Z. Shao, L. Li, Nanostructured polyanilinedecorated Pt/C@PANI core-shell catalyst with enhanced durability and activity, Journal of the American Chemical Society, 134 (2012) 13252-13255.

[27] S. Chaudhari, Y. Sharma, P.S. Archana, Rajan Jose, S. Ramakrishna, S. Mhaisalkar, M. Srinivasan, Electrospun Polyaniline Nanofibers Web Electrodes for Supercapacitors, Journal of Applied Polymer Science, 129 (2013) 1660-1667.

[28] N.J. Pinto, I. Ramos, R. Rojas, P.-C. Wang, A.T. Johnson, Electric response of isolated electrospun polyaniline nanofibers to vapors of aliphatic alcohols, Sensors and Actuators B: Chemical, 129 (2008) 621-627.

[29] Q. Wang, Q. Yao, J. Chang, L. Chen, Enhanced thermoelectric properties of CNT/PANI composite nanofibers by highly orienting the arrangement of polymer chains, Journal of Materials Chemistry, 22 (2012) 17612.

[30] J.B. Ballengee, P.N. Pintauro, Preparation of nanofiber composite proton-exchange membranes from dual fiber electrospun mats, Journal of Membrane Science, 442 (2013) 187-195.

[31] I.D. Norris, M.M. Shaker, F.K. Ko, A.G. MacDiarmid, Electrostatic fabrication of ultrafine conducting fibers: polyanilinerpolyethylene oxide blends, Synthetic Metals, 114 (2000) 109-114.

[32] B. Sundaray, A. Choi, Y.W. Park, Highly conducting electrospun polyaniline-polyethylene oxide nanofibrous membranes filled with single-walled carbon nanotubes, Synthetic Metals, 160 (2010) 984988.

[33] Y. Zhang, G.C. Rutledge, Electrical Conductivity of Electrospun Polyaniline and Polyaniline-Blend Fibers and Mats, Macromolecules, 45 (2012) 4238-4246.

[34] A.G. MacDiarmid, Polyanilines: A Novel Class of Conducting Polymers, Faraday Discuss Chemical Society, 88 (1989) 317-332.

[35] J. Chiang, A.G. Macdiarmid, Polyaniline: Protonic acid doping of the emearaldine form to the metalic regime, Synthetic Metals, 13 (1986) 193-205.

[36] A. MacDiarmid, Synthetic Metals: A Novel Role for Organic Polymers Angewandte Chemie, 40 (2001) 2581-2590.

[37] J.B. Ballengee, P.N. Pintauro, Composite Fuel Cell Membranes from Dual-Nanofiber Electrospun Mats, Macromolecules, 44 (2011) 7307-7314.

[38] G. Li, P.G. Pickup, Ionic Conductivity of PEMFC Electrodes, Journal of The Electrochemical Society, 150 (2003) C745-C752.

[39] T.A. Zawodzinski, C. Derouin, S. Radzinski, Ruth J. Sherman, V.T. Smith, T.E. Springer, S. Gottesfeld, Water Uptake by and Transport Through Nafion-117 Membranes, J. Electrochemical Soc, 140 (1993). 
[40] J. Choi, K.M. Lee, R. Wycisk, P.N. Pintauro, P.T. Mather, Nanofiber composite membranes with low equivalent weight perfluorosulfonic acid polymers, Journal of Materials Chemistry, 20 (2010) 6282.

[41] R. Scigala, A. Wlochowicz, Crystallinity of poly(tetrafluoroethylene), Acta polymerica, 40 (1989) 1518.

[42] L. Grosmaire, S. Castagnoni, P. Huguet, P. Sistat, M. Boucher, P. Bouchard, P. Bebin, S. Deabate, Probing proton dissociation in ionic polymers by means of in situ ATR-FTIR spectroscopy, Physical chemistry chemical physics : PCCP, 10 (2008) 1577-1583.

[43] A. Gruger, A. Regis, T. Schmatko, P. Colomban, Nanostructure of Nafion membranes at different states of hydration an IR and Raman, Vibrational Spectroscopy, 26 215-225.

[44] M. Laporta, M. Pegoraro, L. Zanderighi, PerÑuorosulfonated membrane (Nafion): FT-IR study of the state of water with increasing humidity, Phys. Chem. Chem. Phys., 1 ( 1999) 4619-4628.

[45] F. Zhang, Z. Zhang, Y. Liu, J. Leng, Shape memory properties of electrospun nafion nanofibers, Fibers and Polymers, 15 (2014) 534-539.

[46] B.G. Choi, H. Park, H.S. Im, Y.J. Kim, W.H. Hong, Influence of oxidation state of polyaniline on physicochemical and transport properties of Nafion/polyaniline composite membrane for DMFC, Journal of Membrane Science, 324 (2008) 102-110.

[47] R.A. Huggins, Simple Method to Determine Electronic and lonic components of the conductivity in mixed conductors, lonics, 8 (2002) 301-313.

[48] T. Soboleva, Z. Xie, Z. Shi, E. Tsang, T. Navessin, S. Holdcroft, Investigation of the through-plane impedance technique for evaluation of anisotropy of proton conducting polymer membranes, Journal of Electroanalytical Chemistry, 622 (2008) 145-152.

[49] A.E. Javier, S.N. Patel, D.T. Hallinan, Jr., V. Srinivasan, N.P. Balsara, Simultaneous electronic and ionic conduction in a block copolymer: application in lithium battery electrodes, Angewandte Chemie, 50 (2011) 9848-9851.

[50] Z. Xie, C. Song, B. Andreaus, Titichai Navessin, Z. Shi, J. Zhang, S. Holdcrof, Discrepancies in the Measurement of Ionic Conductivity of PEMs Using Two- and Four-Probe AC Impedance Spectroscopy, Journal of The Electrochemical Society,, 153 (10) (2006) E173-E178. 
Figure 1: Schematic of the dual electrospinning set-up used to fabricate Nafion/PANI composite nanofibers mat.

Figure 2: SEM micrograph of as electrospun Nafion-98 (a) and AN/C Nafion-98 (b)

Figure 3: SEM micrograph of as electrospun PANI-90.

Figure 4: SEM micrograph of as electrospun Nafion-PANI (a) and AN Nafion-PANI (b) AN/C Nafion-PANI (c) and AN/C/T Nafion-PANI (d)

Figure 5: XRD spectrum of Nafion-212 membrane and Nafion-98 nanofiber samples (a), deconvoluted peaks for Nafion-212 membrane (b) and AN Nafion-98 nanofibers (5 mins) (c). Peaks were fitted using Pearson VII distribution function in OriginPro software.

Figure 6: XRD PANI and dual Nafion-PANI nanofibers

Figure 7: FTIR spectra of Nafion, PANI and dual Nafion-PANI nanofibers. 
Figure 8: Nyquist plot of co-electrospun Nafion-PANI and fitted data.

Figure 9: Equivalent circuit used to decouple electrical conductivity from proton conductivity

Figures

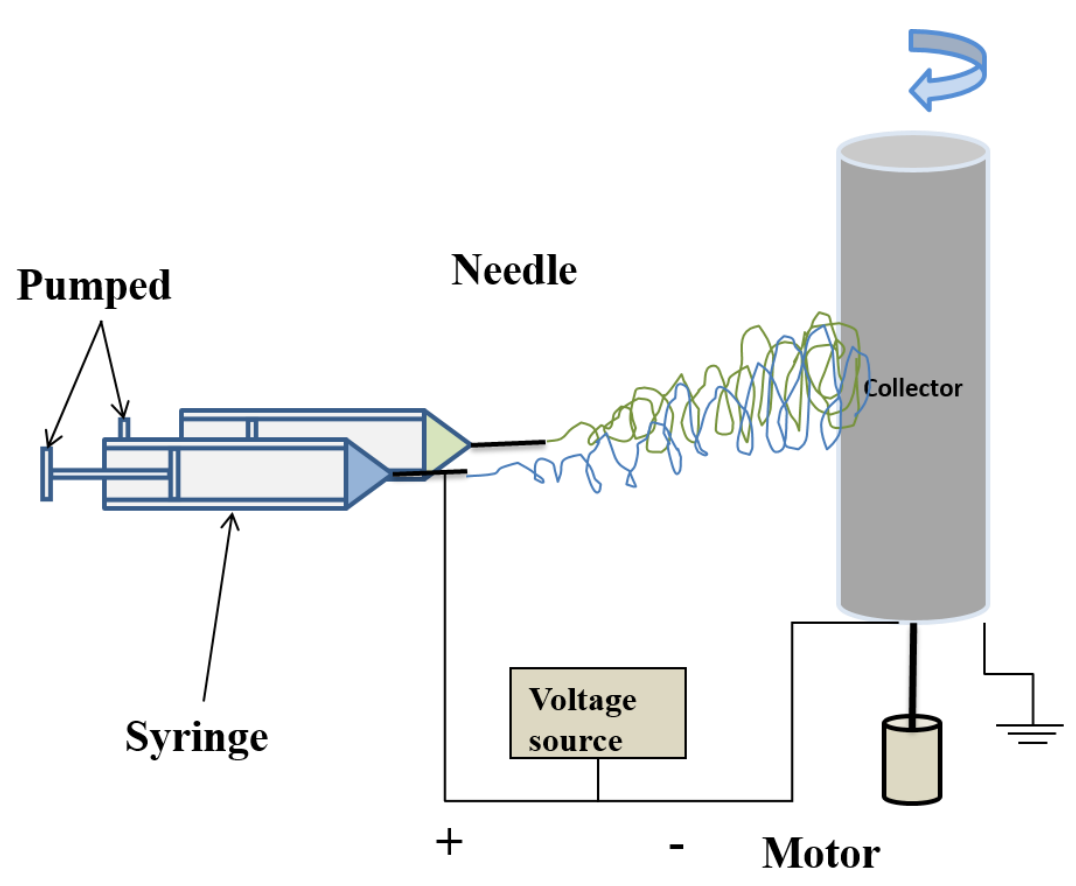

Figure 1 

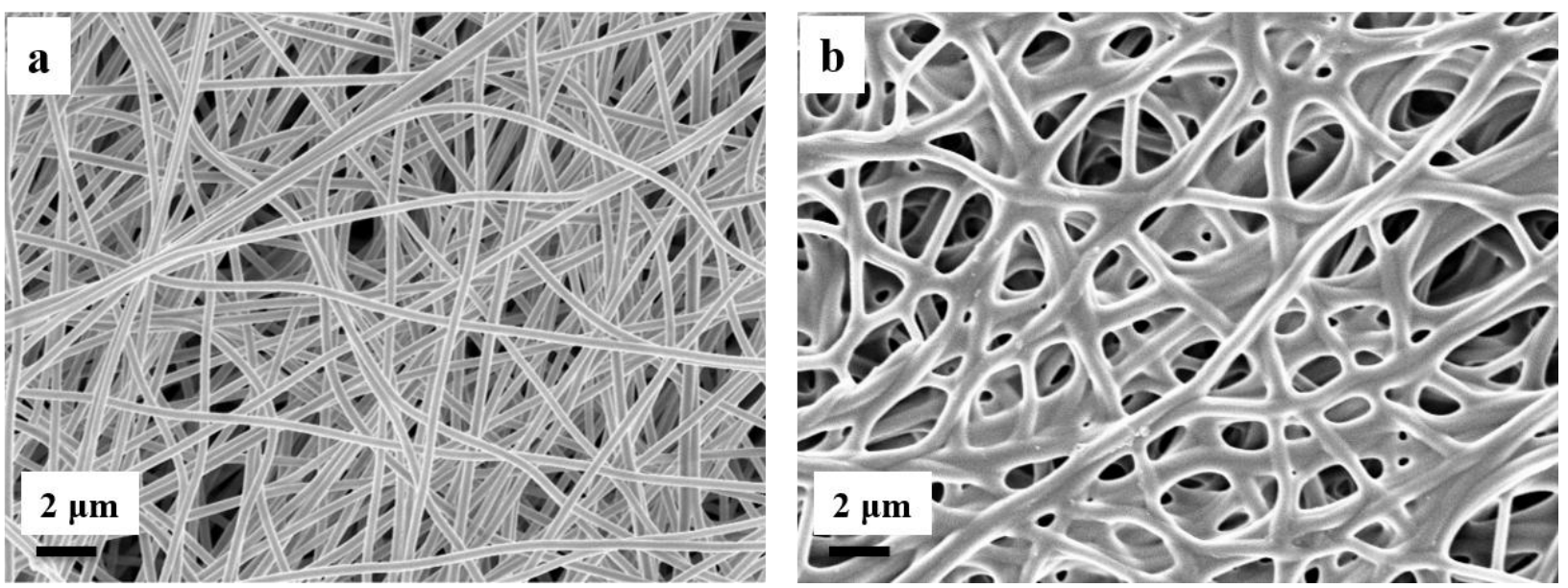

Figure 2
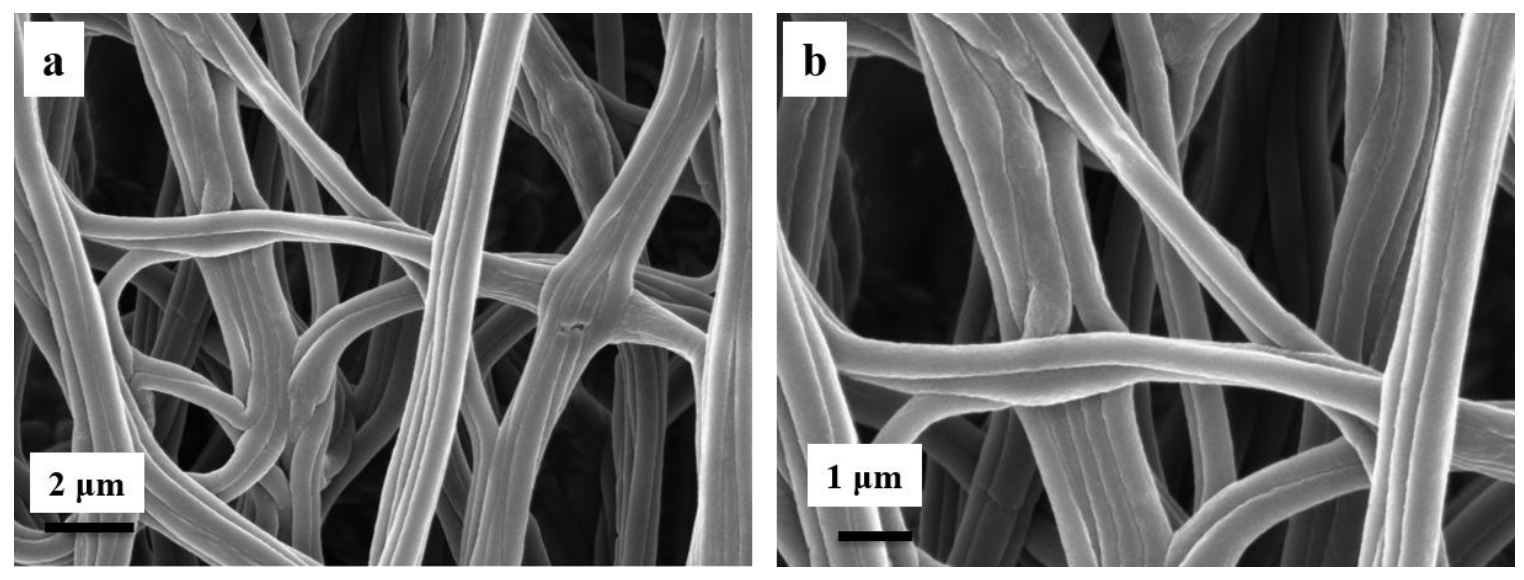

Figure 3 


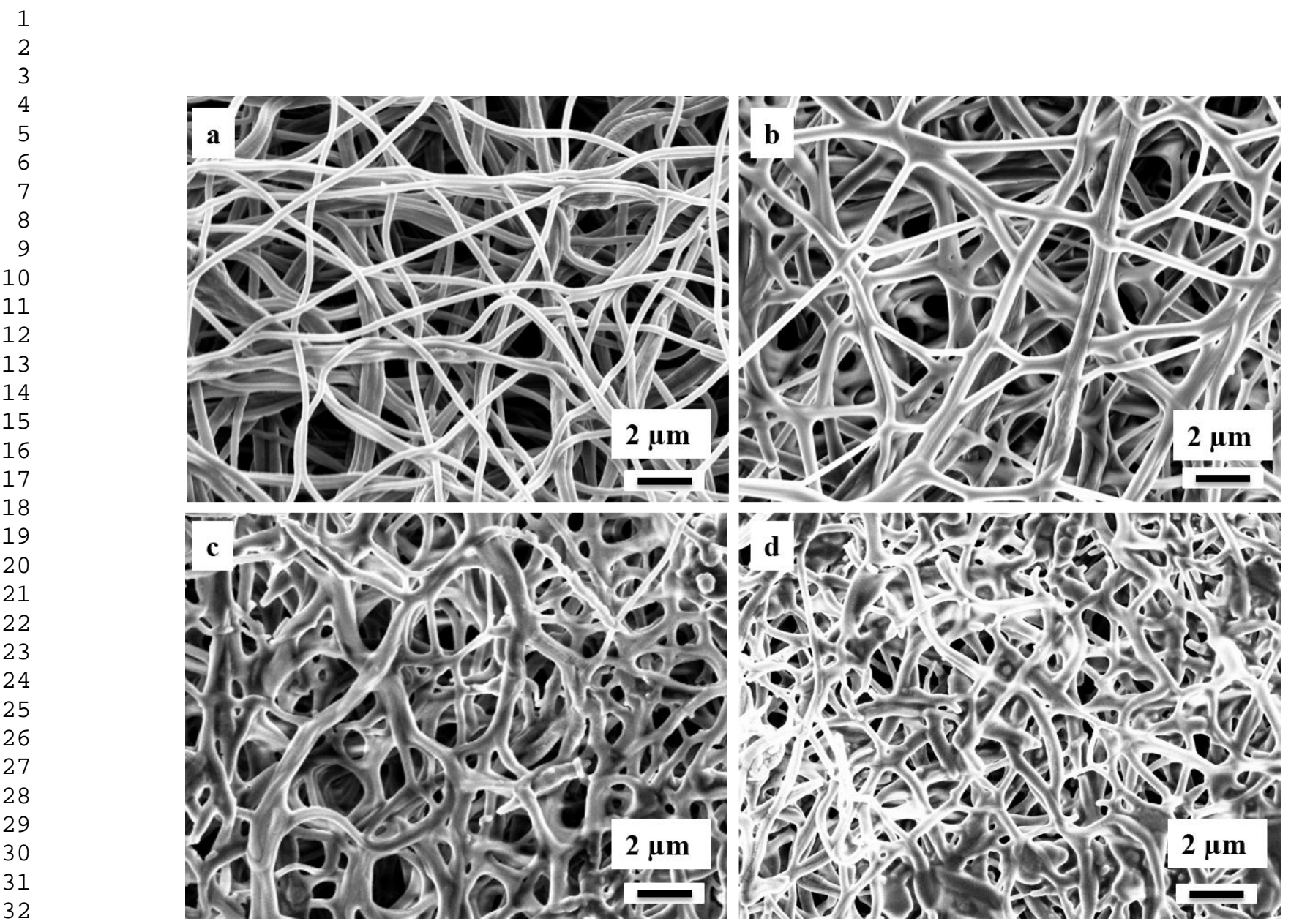

Figure 4 


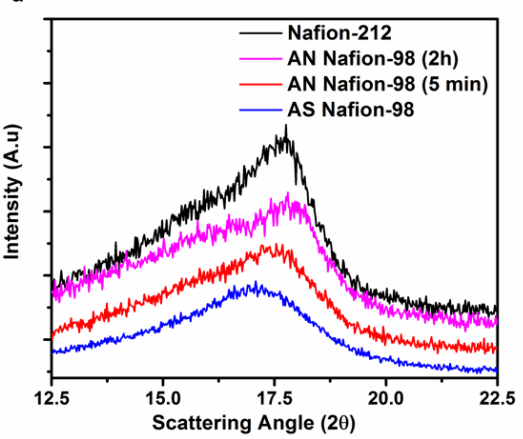

b

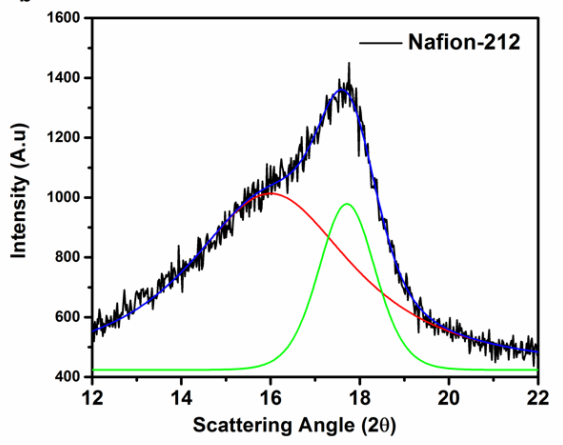

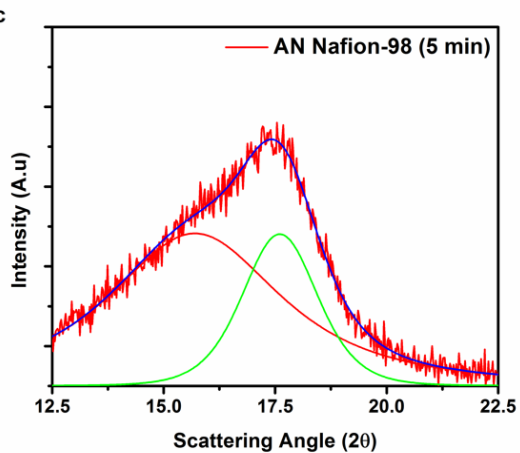

Figure 5

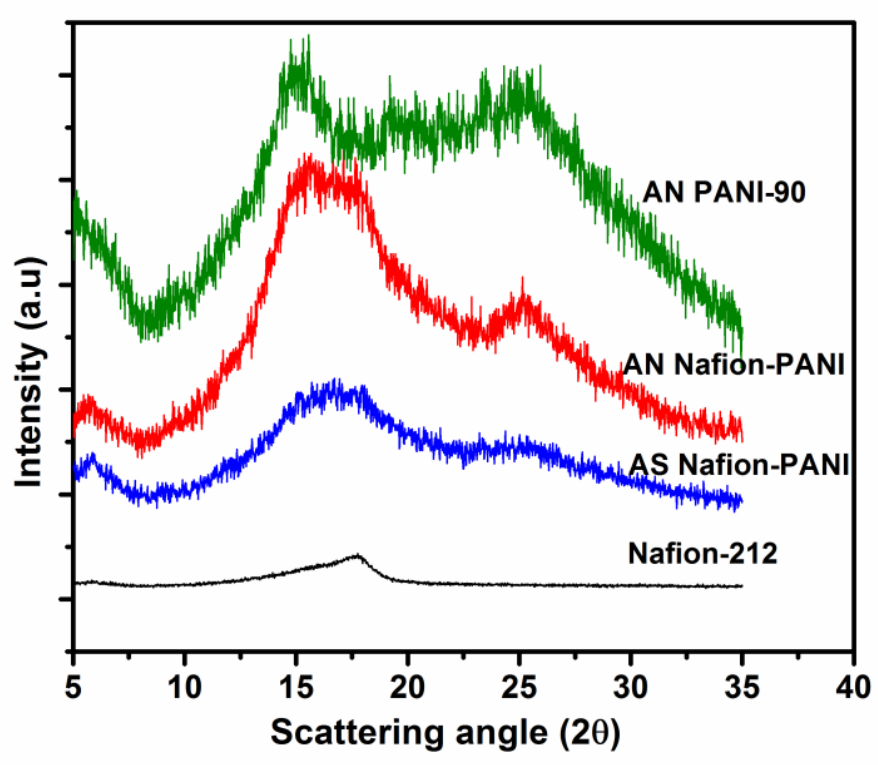

Figure 6 
b
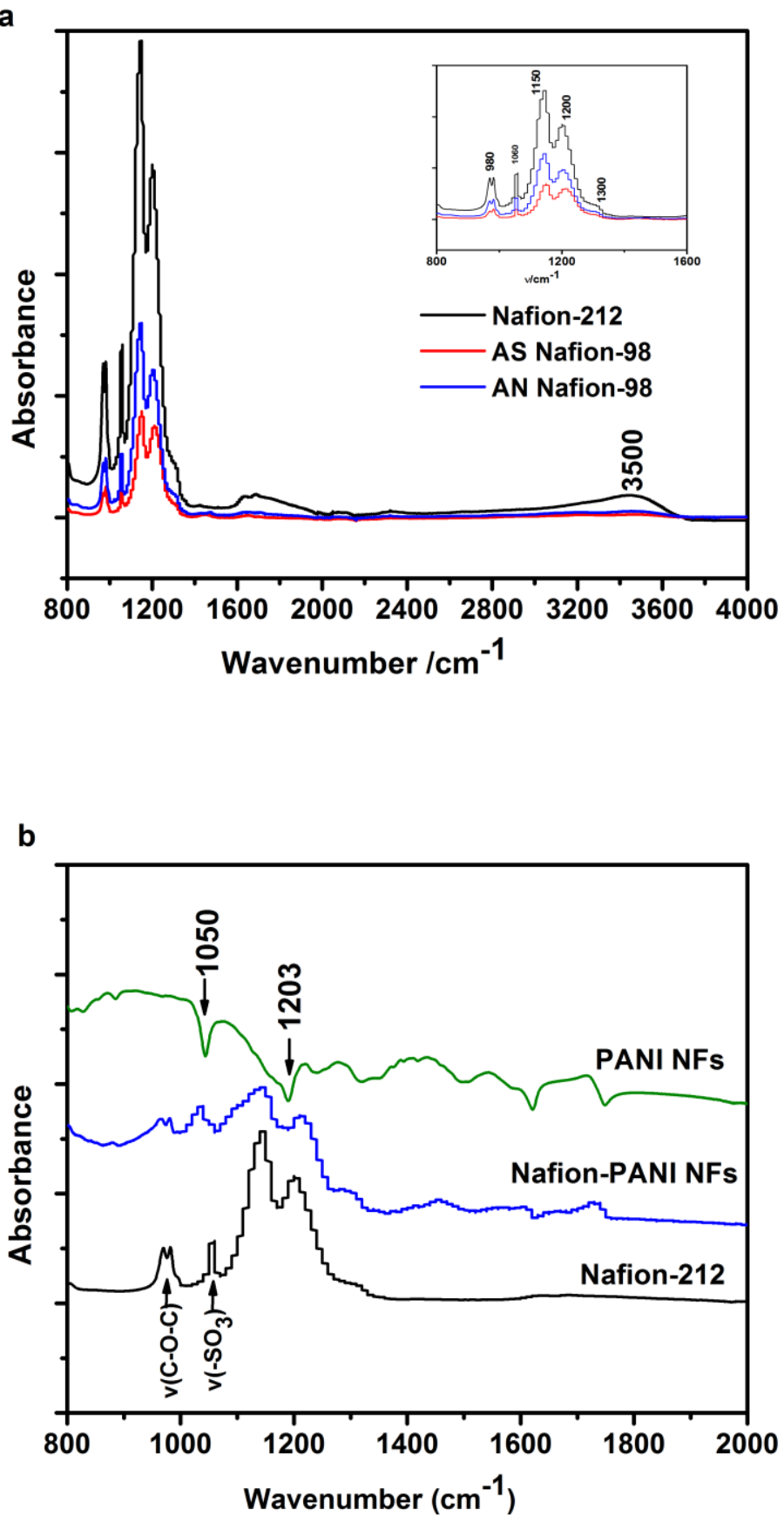

Figure 7 


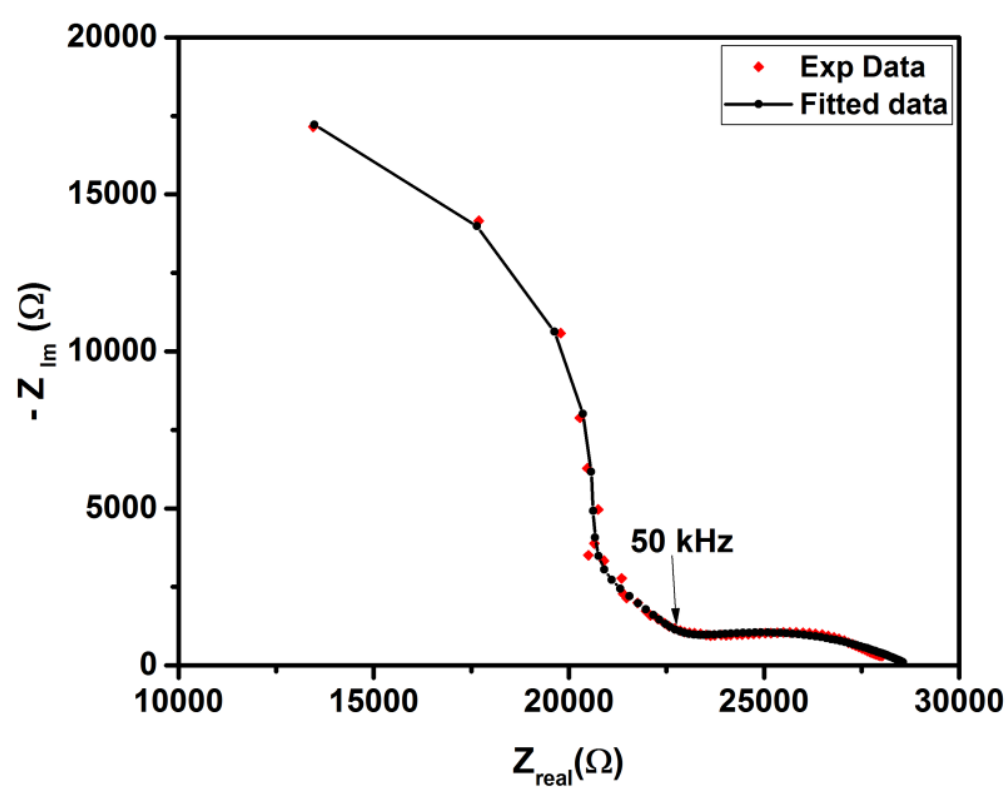

Figure 8

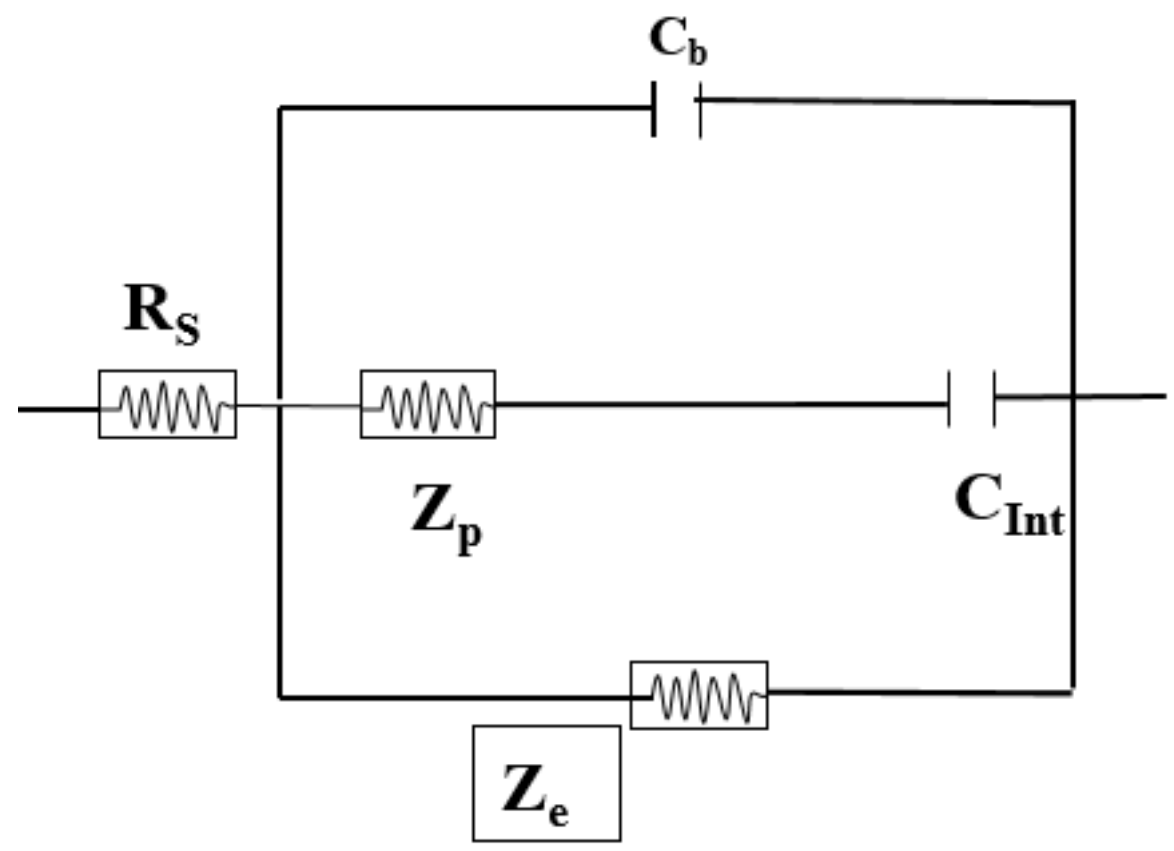

Figure 9 